\title{
BOUNDARY VALUE PROBLEMS FOR MINIMAL SURFACES WITH SINGULARITIES AT INFINITY(1)
}

\author{
BY \\ LIPMAN BERS

\section{Statement OF PROBlems AND RESUlts}

1. Introduction. The extensive literature on boundary value problems for minimal surfaces concerns itself primarily with Plateau's problem and its generalizations. In its simplest form the problem of Plateau is equivalent to the Dirichlet problem for the nonlinear partial differential equation

$$
\left(1+\phi_{y}^{2}\right) \phi_{x x}-2 \phi_{x} \phi_{y} \phi_{x y}+\left(1+\phi_{x}^{2}\right) \phi_{y y}=0 .
$$

In this paper we deal with another kind of boundary value problem for the same equation. We are looking for a solution defined in a domain exterior to a simple closed curve $P$, possessing at the point at infinity a singularity, having on $P$ continuous partial derivatives, and satisfying either of the homogeneous boundary conditions

$$
\mathfrak{A}: \quad \partial \phi / \partial n=0, \quad \mathfrak{B}: \quad \phi=0 \text { on } \mathbb{P} \text {. }
$$

We shall not require, in general, that $\phi(x, y)$ be single-valued, but shall assume that

$$
w=\phi_{x}-i \phi_{y}
$$

is. We shall also prescribe the value of

$$
q_{\max }=\max |w| \text {. }
$$

It will be convenient to associate with every solution of (1.1) the func$\cdot \operatorname{tion}\left({ }^{2}\right)$

$$
\psi(x, y)=\int \frac{\phi_{x} d y-\phi_{y} d x}{\left(1+\phi_{x}^{2}+\phi_{y}^{2}\right)^{1 / 2}} .
$$

It is clear that the boundary condition $\mathfrak{A}$ may be written as

$$
\mathfrak{A}: \quad \psi=0 \text { on } P \text {. }
$$

Presented to the Society, April 29, 1949; received by the editors December 24, 1949.

(1) Part of the research leading to this paper was sponsored by the Office of Naval Research (Contract N6-onr-248). A partial result was announced in [4]. Numbers in brackets refer to the references at the end of the paper.

(2) The line integral in (1.5) is path-independent by virtue of (1.1). The function $\psi$ is determined but for an additive constant. 
The singularity of $\phi$ at infinity must be of a rather special nature. In fact, we have shown $\left({ }^{3}\right)$ that under the conditions stated above $\phi$ admits the asymptotic formula

$$
\begin{aligned}
& \phi(x, y)=A x+B y+C \log \left(x^{2}+y^{2}\right)^{1 / 2}+D \arctan (y / x)+R(x, y), \\
& R(x, y)=O(1), \quad R_{x}(x, y), \quad R_{y}(x, y) \rightarrow 0 \quad \text { as } x^{2}+y^{2} \rightarrow \infty .
\end{aligned}
$$

It is clear that condition $\mathfrak{B}$ implies that $D=0$, and it is easy to see that $\mathfrak{A}$ implies that $C=0$.

Chaplygin $\left.{ }^{4}\right)$ noticed that equation (1.1) may be interpreted as the potential equation of a steady gas flow (with stream-function $\psi$ and conjugate complex velocity $w$ ). The density $\rho$ of a Chaplygin flow is connected with the speed $q=|w|$ by the relation

$$
\rho^{2}\left(1+q^{2}\right)=1
$$

which would imply the (physically impossible) pressure-density relation $p=A+B / \rho$. It turns out that Chaplygin flows are good approximations to sufficiently slow flows of an actual gas.

Our boundary value problems were suggested by this gas-dynamical interpretation of equation (1.1). The same interpretation also suggests, by analogy with classical hydrodynamics, the method of solution.

(Note that solutions of partial differential equations satisfying homogeneous boundary conditions and possessing prescribed singularities (for example Green's and Neumann's functions) are of importance in the case of linear equations of elliptic type. There, however, no separate theory is needed, since singularities may be removed by subtracting appropriate particular solutions.)

2. The boundary value problem of airfoil theory. We shall consider in detail the following problem $\left.{ }^{5}\right)$.

PROBLEM $\mathbf{A}_{1}$. The profile $\mathbb{P}$ has no protruding corners or cusps, except perhaps one at the point $z_{T}$. The unknown function $\phi(x, y)$ has to satisfy condition $\mathfrak{A}$, all conditions stated in $\$ 1$, and, in addition, the following. The limit

$$
w_{\infty}=\lim _{x^{2}+y^{2} \rightarrow \infty}\left(\phi_{x}-i \phi_{y}\right)
$$

(which exists and is finite by virtue of (1.7)) is positive. At $z_{T}$ the curve $\psi=0$ divides itself into two branches.

In gas-dynamical language the problem may be stated as that of de-

(3) See [5]. We note, however, that the present paper is self-contained.

(4) [6]. An English translation of this paper was published by the National Advisory Committee for Aeronautics (Technical Memorandum 1063).

(5) A modified form of this problem (with $\left|w_{\infty}\right|$ being prescribed instead of $q_{\max }$ ) was considered by Frankl and Keldysh [8] for a more general equation. These authors obtain an existence and uniqueness theorem for sufficiently small values of $\left|w_{\infty}\right|$. 
termining a steady Chaplygin flow past a given profile $P$, satisfying at $z_{T}$ the Kutta-Joukowsky condition, possessing a prescribed direction at infinity, and (by virtue of (1.4)) possessing a prescribed maximum local speed.

To every problem with condition $\mathfrak{A}$ there corresponds a "dual" one involving the same restrictions on the profile and condition $\mathfrak{B}$.

Problem $\mathrm{B}_{1} . z_{T}$ is a branch-point of the curve $\psi=0 . w_{\infty}$ is positive.

It will be seen that the method used for solving Problem $A_{1}$ is applicable also to $\mathbf{B}_{1}$ as well as to all other problems considered. For this reason we will give proofs for the case $\mathbf{A}_{1}$ only.

3. Other boundary value problems. In the problems to be stated now the profile is assumed to have no protruding corners or cusps.

Problem $\mathbf{A}_{2} . \phi$ is single-valued. $w_{\infty}$ is positive.

Gas-dynamical interpretation: to find a circulation-free flow past $P$. Geometrical interpretation: to find a one-sheeted minimal surface extending to infinity, intersecting a given cylinder orthogonally, and possessing at infinity a tangent plane containing a given direction (orthogonal to the directrices of the cylinder $)\left({ }^{6}\right)$.

Problem $\mathrm{B}_{2} . \psi$ is single-valued. $w_{\infty}$ is positive.

Geometrical interpretation: to find a minimal surface bounded by a plane curve, extending to infinity and possessing there a tangent plane which contains a given direction (parallel to the plane of the boundary curve).

Problem $\mathbf{A}_{3} . w_{\infty}=0$.

Gas-dynamical interpretation: to find a purely circulatory flow past $P$. Geometrical interpretation: to find a minimal surface which winds itself around a cylinder, intersects the cylinder orthogonally, and possesses at infinity a tangent plane orthogonal to the directrices of the cylinder.

Problem $\mathbf{B}_{3} . w_{\infty}=0$.

Geometrical interpretation: to find a minimal surface bounded by a plane curve and possessing at infinity a tangent plane parallel to the plane of the curve.

Some other problems are considered in Chapter IV.

4. Conditions on the profile. We shall require that the curve $P$ possess a continuously turning tangent (except perhaps at $z_{T}$ ). Without loss of generality we assume that the length of $P$ is $2 \pi$. Then $P$ admits the parametric representation

( ${ }^{6}$ A modified form of Problem $A_{2}$ (cf. §19) was treated by Slioskin [15] for the case of a symmetrical profile $P$. Slioskin prescribes the value of $\left|w_{\infty}\right|$ and reduces the problem to an integro-differential equation which is different from the one used here. The same modification of $\mathbf{A}_{2}$ has been attacked by Chen [16] by variational methods. He shows that a certain variational problem related to the boundary value problem has a solution. Whether this solution also solves the boundary value problem remains an open question.

Here and hereafter the term "tangent plane at infinity" refers merely to the limiting position of the surface normal. 


$$
\begin{array}{r}
x+i y=z=Z(s)=z_{T}+\int_{0}^{s} e^{i \theta(\sigma)} d \sigma, \quad 0 \leqq s \leqq 2 \pi, \\
Z(2 \pi)=z_{T}, \quad Z(s) \neq Z\left(s^{\prime}\right) \quad \text { for } 0 \leqq s<s^{\prime}<2 \pi .
\end{array}
$$

We make the following assumption concerning the function $\Theta(s)$.

$1^{\circ}$. Set $\Theta(2 \pi)-\Theta(0)=(1+a) \pi$. Then $a=1$, except in Problems $\mathbf{A}_{1}, \mathbf{B}_{1}$ where $0 \leqq a \leqq 1$.

$2^{\circ}$. $\Theta(s)$ satisfies a uniform Hölder condition, that is, there exist positive constants $K, k$, such that

$$
\left|\Theta(s)-\Theta\left(s^{\prime}\right)\right| \leqq K\left|s-s^{\prime}\right|^{k} \quad \text { for } 0 \leqq s \leqq s^{\prime} \leqq 2 \pi .
$$

$3^{\circ}$. $\Theta(s)$ is absolutely continuous and

$$
\int_{0}^{2 \pi}\left|\min \left\{\Theta^{\prime}(s), 0\right\}\right| d s<\pi
$$

The last inequality expresses the "essential convexity" of $P$; along the arcs of negative curvature the tangent is turned by less than $\pi$.

It can be shown that the condition on the absence of convex corners (except one in Problems $\mathbf{A}_{1}, \mathbf{B}_{1}$ ) is necessary for the existence of solutions $\left({ }^{7}\right)$. We do not know whether the condition on essential convexity is.

Our main result may now be stated.

EXISTENCE THEOREM. Under the conditions on $P$ stated above the problems $\mathrm{A}_{1}-\mathrm{B}_{3}$ have solutions.

In $\$ 18$ we shall show that the existence theorem remains valid for essentially convex profiles possessing a finite number of intruding corners.

5. Outline of the existence proof. Assume for the moment that we want to solve one of our problems, say $\mathbf{A}_{1}$, for the Laplace equation (in hydrodynamical terms: for an incompressible fluid). It is well known that the solution can be obtained by conformal mapping. The functions $\phi$ and $\psi$ must now be conjugate harmonic, that is,

$$
F=\phi+i \psi
$$

must be an analytic function of

$$
z=x+i y .
$$

If we map conformally the domain $\varepsilon(P)$ exterior to $P$ onto $|\zeta|>1$ taking $z=\infty$ into $\zeta=\infty, z=z_{T}$ into $\zeta=1$, then $F$ must be of the form

$$
F(\zeta)=C e^{-i \tau}\left(\zeta+\frac{e^{2 i \tau}}{\zeta}+2 i e^{i \tau} \sin \tau \log \zeta\right)
$$

(7) The proof, stated in gas-dynamical language, will be found in [4]. 
(where $C>0$ and $\tau$ is real). The problem will be solved once we know the analytic function mapping $|\zeta|>1$ onto $\mathcal{E}(P)$. Since the conjugate complex velocity $w$ (defined by (1.3)) is an analytic function of $z$ and hence of $\zeta$, and

$$
w=d F / d z=(d F / d \zeta)(d \zeta / d z),
$$

the desired mapping function will have the form

$$
z=\int \frac{F^{\prime}(\zeta)}{w} d \zeta
$$

A conformal mapping, however, is known, once we find the one-to-one correspondence between the boundaries. In our case this correspondence will be described by a function $s=f(\omega)$ such that (5.4) takes $\zeta=e^{i \omega}$ into $z=Z(s)$. Noting (4.1), we may write the mapping (5.4) on the circle $|\zeta|=1$ in the form

$$
\int_{0}^{f(\omega)} e^{i \Theta(\sigma)} d \sigma=i \int_{0}^{\omega} \frac{F^{\prime}\left(e^{i \sigma}\right) e^{i \sigma} d \sigma}{w\left(e^{i \sigma}\right)} .
$$

Differentiating with respect to $\omega$ and solving for $w$, we obtain

$$
w\left(e^{i \omega}\right)=i e^{-i \Theta[f(\omega)]+i \omega} F^{\prime}\left(e^{i \omega}\right) / f^{\prime}(\omega) .
$$

The requirement that the right-hand side of (5.6) be the boundary function of a regular analytic function leads to an integral equation for the function $f(\omega)$.

An analogous method may be applied to equation (1.1). We assume the existence of a solution of $A_{1}$ and map $\varepsilon(P)$ conformally with respect to the metric

$$
d S^{2}=d x^{2}+d y^{2}+d \phi^{2}
$$

onto $|\zeta|>1\left(^{8}\right)$. Then $F$ (defined by (5.1)) becomes an analytic function of $\zeta$, and if the mapping is normalized as before $F(\zeta)$ must be of the form (5.3). While $w$ will not be an analytic function of $\zeta$, the function

$$
w^{*}=\frac{\phi_{x}-i \phi_{y}}{1+\left(1+\phi_{x}^{2}+\phi_{y}^{2}\right)^{1 / 2}}
$$

will, and the mapping of $|\zeta|>1$ onto $\varepsilon(P)$ will be given by the formula

$$
z=\frac{1}{2} \int \frac{F^{\prime}(\zeta)}{w^{*}} d \zeta-\frac{1}{2} \int \overline{F^{\prime}(\zeta)} \overline{w^{*}} \overline{d \zeta}
$$

(8) This mapping is equivalent to the mapping of the "physical" $(x, y)$ plane onto the "hodograph plane" $\left(\left(\phi_{x}, \phi_{y}\right)\right.$ plane) followed by a distortion of the hodograph plane according to (5.8) and by a (uniformizing) conformal mapping of the distorted hodograph plane into the $\zeta$-plane. 
On the boundary this may be written in the form

$$
\int_{0}^{f(\omega)} e^{i \Theta(\sigma)} d \sigma=\frac{i}{2} \int_{0}^{\omega}\left\{\frac{F^{\prime}\left(e^{i \sigma}\right)}{w^{*}\left(e^{i \sigma}\right)} e^{i \sigma}+\overline{F^{\prime}\left(e^{i \sigma}\right)} \overline{w^{*}\left(e^{i \sigma}\right)} e^{-i \sigma}\right\} d \sigma .
$$

Differentiating with respect to $\omega$, we obtain a relation from which $w^{*}\left(e^{i \omega}\right)$ may be determined in terms of $f(\omega)$. The condition that $w^{*}\left(e^{i \omega}\right)$ be the boundary function of a regular analytic function leads to a nonlinear integral equation for $f(\omega)\left({ }^{9}\right)$. It can be shown that, conversely, a solution of the integral equation leads to a solution of the original boundary value problem.

The derivation of the integral equation is given in Chapter II. In Chapter III we complete the existence proof by showing that the integral equation has a solution. This is accomplished by means of the powerful topological method due to Leray and Schauder. As an essential tool in verifying the conditions of the Schauder-Leray theorem we use the theory of conjugate functions. Chapter IV contains various extensions.

\section{Derivation of THE INTEgRAL EQUATION}

6. The mapping. In this and the following sections we assume that a solution of Problem $\mathbf{A}_{1}$ is given. In the domain $\mathcal{D}=\mathcal{E}(P)-\{\infty\}$ we introduce the metric (5.7). Since

$$
d S^{2}=\left(1+\underset{\phi_{x}^{2}}{2}\right) d x^{2}+2 \phi_{x} \phi_{y} d x d y+\left(1+\phi_{y}^{2}\right) d y^{2},
$$

this is a Riemann metric. The general uniformization theorem $\left({ }^{10}\right)$ assures the existence of a homeomorphism

$$
\xi=\xi(x, y), \quad \eta=\eta(x, y)
$$

of $\mathcal{D}$ onto a (doubly connected) simply covered domain $\Delta$ of the $\zeta$-plane $(\zeta=\xi+i \eta)$ which is conformal with respect to the metric (6.1), that is, such that $\xi$ and $\eta$ are continuously differentiable and

$$
|d \zeta|^{2}=p(x, y) d S^{2}, \quad p>0, \quad \xi_{x} \eta_{y}-\xi_{y} \eta_{x}>0 .
$$

A direct computation( $\left.{ }^{11}\right)$ verifies that $F$ and $w^{*}$ are regular analytic functions of $\zeta$, that $w^{*}(\zeta)$ and $F^{\prime}(\zeta)$ are single-valued, and that throughout $\Delta$

$$
F^{\prime}(\zeta) / w^{*}(\zeta) \neq 0, \infty \text {. }
$$

We also note that (5.8) implies the inequality

$$
\left|w^{*}(\zeta)\right|<1
$$

(9) A slightly modified form of these integral equations was given in [2] and [3]. In these papers the emphasis was on finding numerical solutions. For a different approach to this problem by Gelbart, Bartnoff, and Resh see $[1 ; 9 ; 10]$.

(10) See, for instance, $[11$, p. 476$]$.

(11) The details of this computation will be found in [5]. 
The boundary continuum $\Gamma_{\infty}$ of $\Delta$ corresponding to $z=x+i y=\infty$ is a point. In fact, assume that $\Gamma_{\infty}$ is not degenerate. By virtue of (2.1) and (5.8), w* is constant on $\Gamma_{\infty}$, hence constant in $\Delta$. It follows that $w$ is constant and $\phi(x, y)$ linear, which is plainly impossible. The boundary continuum $\Gamma_{0}$ of $\Delta$ corresponding to $P$ is nondegenerate, for otherwise $w^{*}(\zeta)$ would be regular for all values of $\zeta$, which is impossible. Since $\Delta$ is determined but for a conformal transformation, we may assume that $\Gamma_{\infty}$ is the point $\zeta=\infty$ and $\Gamma_{0}$ the circle $|\zeta|=1$

A direct computation yields the relation $\left({ }^{12}\right)$

$$
d z=\frac{1}{2}\left(\frac{d F}{w^{*}}-\bar{w}^{*} \overline{d F}\right)
$$

which shows that the mapping of $|\zeta|>1$ onto $\mathcal{E}(P)$ inverse to (6.2) is given by (5.9).

We make now an assumption (to be justified by the subsequent existence proof).

Assumption $\alpha$. The mapping (5.9) of $|\zeta|>1$ onto $\mathcal{E}(P)$ is a homeomorphism on $|\zeta|=1$.

Hence there exists a function $s=f(\omega)$ such that $\zeta=e^{i \omega}$ is taken by (5.9) into $z=Z(s)$. We may assume that $\zeta=1$ is taken into $z_{T}$. Then

$$
f(0)=0, \quad f(2 \pi)=2 \pi .
$$

The function $f$ is continuous and strictly increasing.

Assumption 3 . The function $f(\omega)$ is continuously differentiable.

This assumption, as well as the one to be made later, will be justified by the existence proof.

7. The functions $F$ and $w^{*}$ in the $\zeta$-plane. The function $F(\zeta)$ is such that Im $F\left(e^{i \omega}\right)=0$. This follows at once from boundary condition $\mathfrak{A}$, and implies that $F(\zeta)$ is regular for $|\zeta|=1$. On the other hand $\zeta=1$ is a branch-point of a curve $\operatorname{Im} F=0$ so that $F^{\prime}(1)=0$. We claim that $F^{\prime}(\zeta)$ is regular at $\zeta=\infty$ and that $F^{\prime}(\infty) \neq 0$.

In fact, $w^{*}(\zeta)$ is regular at $\zeta=\infty$ by virtue of (6.4), and from the conditions of Problem $\mathbf{A}_{1}$ it follows that $w^{*}(\infty)>0$. We write (5.9) in the form

$$
x=\operatorname{Re} G_{1}(\zeta), \quad y=\operatorname{Re} G_{2}(\zeta)
$$

where

$$
\begin{aligned}
& G_{1}(\zeta)=\frac{1}{2} \int F^{\prime}(\zeta)\left(\frac{1}{w^{*}}-w^{*}\right) d \zeta \\
& G_{2}(\zeta)=-\frac{i}{2} \int F^{\prime}(\zeta)\left(\frac{1}{w^{*}}+w^{*}\right) d \zeta
\end{aligned}
$$

(12) This relation is due to Chaplygin [6]. 
From the fact that (7.1) maps a neighborhood of $\zeta=\infty$ in a one-to-one way onto a neighborhood of $z=\infty$ we may conclude( $\left.{ }^{13}\right)$ that $G_{1}^{\prime}(\zeta)$ and $G_{2}^{\prime}(\zeta)$ are regular at $\zeta=\infty$ and that $G_{1}^{\prime}(\infty) \neq 0, G_{2}^{\prime}(\infty) \neq 0$. From this the assertion on $F^{\prime}(\infty)$ follows.

The above properties of $F(\zeta)$ determine this function uniquely, except for three constant parameters. We see that $F(\zeta)$ differs from (5.3) by at most a real additive constant. Thus

$$
F^{\prime}(\zeta)=C e^{-i \tau}\left(1-\frac{1}{\zeta}\right)\left(1+\frac{e^{2 i \tau}}{\zeta}\right)
$$

where $C>0$ and $\tau$ is real.

By (7.3) and (6.3) we see that $w^{*}(\zeta) \neq 0$ for $|\zeta|>1$. From the assumptions made concerning the mapping and from the requirement that $w$ be continuous on $P$ we conclude that $w^{*}(\zeta)$ is continuous for $|\zeta|=1$. It is clear that $w^{*}$ must vanish at $\zeta=1$ and at $\zeta=-e^{2 i \tau}$, since $F^{\prime} / w^{*}$ must be integrable at these points. We assume (Assumption $\gamma)$ that $w^{*}\left(e^{i \omega}\right) \neq 0$ for $\omega \neq \equiv 0,2 \tau+\pi(\bmod 2 \pi)$.

The mapping of $|\zeta|=1$ onto $P$ is given by (5.10). Differentiating both sides with respect to $\omega$ and noting that

$$
F^{\prime}\left(e^{i \omega}\right)=4 C i e^{-i \omega} \sin \frac{\omega}{2} \cos \left(\frac{\omega}{2}-\tau\right)
$$

we obtain

$$
f^{\prime}(\omega)=-2 C \sin \frac{\omega}{2} \cos \left(\frac{\omega}{2}-\tau\right)\left\{\frac{1}{q^{*}(\omega)}-q^{*}(\omega)\right\} e^{i\{\theta(\omega)-\Theta[f(\omega)]\}},
$$

where we set

$$
w^{*}\left(e^{i \omega}\right)=q^{*}(\omega) e^{-i \theta(\omega)}
$$

Thus

$$
\theta(\omega) \equiv\left\{\begin{array}{ll}
\Theta[f(\omega)]+\pi, & 0<\omega<2 \tau+\pi, \\
\Theta[f(\omega)], & 2 \tau+\pi<\omega<2 \pi,
\end{array}(\bmod 2 \pi)\right.
$$

and

$$
f^{\prime}(\omega)=2 C\left|\sin \frac{\omega}{2} \cos \left(\frac{\omega}{2}-\tau\right)\right|\left\{\frac{1}{q^{*}(\omega)}-q^{*}(\omega)\right\} .
$$

Recalling Property $1^{\circ}$ of the function $\Theta(s)$, we conclude from (7.7) that

$$
w^{*}(\zeta)\left(1-\frac{1}{\zeta}\right)^{-a}\left(1+\frac{e^{2 i \tau}}{\zeta}\right)^{-1}
$$

(13) The details are given in [5]. 
is regular for $|\zeta|>1$, continuous and different from zero for $|\zeta| \geqq 1$. It follows that $w^{*}(\zeta)$ admits the representation

$$
w^{*}(\zeta)=\lambda\left(1-\frac{1}{\zeta}\right)^{a}\left(1+\frac{e^{2 i r}}{\zeta}\right) e^{x(\zeta)}
$$

where $\chi(\zeta)$ is regular for $|\zeta|>1$, continuous on $|\zeta|=1, \chi(\infty) \equiv 0(\bmod 2 \pi i)$, and $\lambda>0$. (By $(1-1 / \zeta)^{a}$ we understand that single-valued branch of the function which equals 1 at $\zeta=\infty$.)

8. The integral equation for Problem $\mathbf{A}_{1}$. Consider the function $\chi(\zeta)$ given by (7.9) and set

$$
\chi\left(e^{i \omega}\right)=h(\omega)+i \tilde{h}(\omega)
$$

then

$$
\frac{1}{2 \pi} \int_{0}^{2 \pi} h(\omega) d \omega=0, \quad \frac{1}{2 \pi} \int_{0}^{2 \pi} \tilde{h}(\omega) d \omega \equiv 0(\bmod 2 \pi) .
$$

The first equation (8.2) shows that

$$
h(\omega)=-\frac{1}{2 \pi} \int_{0}^{\pi}\{\tilde{h}(\omega+t)-\tilde{h}(\omega-t)\} \cot \frac{t}{2} d t .
$$

Conversely, if $h$ is given by (8.3), then (8.1) is the boundary function of an analytic function $\chi(\zeta)$ regular for $|\zeta|>1$, and $\operatorname{Re} \chi(\infty)=0$.

From (7.6), (7.9), and (8.1) we have that

$$
q^{*}(\omega) e^{-i \theta(\omega)}=\lambda\left(1-e^{-i \omega}\right)^{a}\left(1+e^{2 i \tau-i \omega}\right) \dot{e}^{h(\omega)+i \tilde{h}(\omega)},
$$

so that by (7.7)

$$
\tilde{h}(\omega) \equiv-\Theta[f(\omega)]+\frac{1+a}{2} \omega-\left(1+\frac{a}{2}\right) \pi-\tau \quad(\bmod 2) .
$$

The second equation (8.2) now yields a condition determining $\tau$ once $f(\omega)$ is known:

$$
\tau . \equiv \frac{3 \pi}{2}-\frac{1}{2 \pi} \int_{0}^{2 \pi} \Theta[f(\omega)] d \omega
$$

$(\bmod 2 \pi)$

We shall associate to every continuous function $f(\omega)$ satisfying (6.6) the function $\Lambda_{f}(\omega)$ defined by

$$
\begin{aligned}
\Lambda_{f}(\omega) & =\Theta[f(\omega)]-\frac{1+a}{2} \omega, \quad 0 \leqq \omega \leqq 2 \pi, \\
\Lambda_{f}(\omega+2 \pi) & =\Lambda_{f}(\omega) .
\end{aligned}
$$

It follows by (8.3) and (8.5) that 


$$
h(\omega)=\frac{1}{2 \pi} \int_{0}^{\pi}\left\{\Lambda_{f}(\omega+t)-\Lambda_{f}(\omega-t)\right\} \cot \frac{t}{2} d t
$$

or, using the notation standard in the theory of conjugate functions $\left({ }^{14}\right)$,

$$
h=-\bar{\Lambda}_{f}
$$

We also have by (7.6) that

$$
q^{*}(\omega)=2^{1+a} \lambda\left|\sin \frac{\omega}{2}\right|^{a}\left|\cos \left(\frac{\omega}{2}-\tau\right)\right| e^{h(\omega)} .
$$

Since $|w|$ must attain the maximum $q_{\max }$, the maximum of $\left|w^{*}(\zeta)\right|$, and hence that of $q^{*}(\omega)$, must be

$$
\mu=\frac{q_{\max }}{1+\left(1+q_{\max }^{2}\right)^{1 / 2}} .
$$

Thus we obtain from (8.10) an equation for determining $\lambda$ :

$$
\lambda=\mu / 2^{1+a} \max \left\{\left|\sin \frac{\omega}{2}\right|^{a}\left|\cos \left(\frac{\omega}{2}-\tau\right)\right| e^{h(\omega)}\right\} .
$$

Next, substituting (8.10) into (7.8) and integrating we obtain that

$$
\begin{aligned}
f(\omega)=\frac{C}{2^{a} \lambda} \int_{0}^{\omega}\left|\sin \frac{\sigma}{2}\right|^{1-a}\left\{e^{-h(\sigma)}\right. & \\
& \left.-4^{1+a} \lambda^{2}\left|\sin \frac{\sigma}{2}\right|^{2 a} \cos ^{2}\left(\frac{\sigma}{2}-\tau\right) e^{h(\sigma)}\right\} d \sigma
\end{aligned}
$$

and, since $f(2 \pi)=2 \pi$,

$$
\begin{aligned}
C=2^{1-a} \pi \lambda / \int_{0}^{2 \pi}\left|\sin \frac{\sigma}{2}\right|^{1-a}\left\{e^{-h(\sigma)}\right. \\
\\
\left.\quad-4^{1+a} \lambda^{2}\left|\sin \frac{\sigma}{2}\right|^{2 a} \cos ^{2}\left(\frac{\sigma}{2}-\tau\right) e^{h(\sigma)}\right\} d \sigma .
\end{aligned}
$$

Substituting this value of $C$ into (8.13) we get

$$
f(\omega)=\frac{\int_{0}^{\omega}\left|\sin \frac{\sigma}{2}\right|^{1-a}\left\{e^{-h(\sigma)}-4^{1+a} \lambda^{2}\left|\sin \frac{\sigma}{2}\right|^{2 a} \cos ^{2}\left(\frac{\sigma}{2}-\tau\right) e^{h(\sigma)}\right\} d \sigma}{\frac{1}{2 \pi} \int_{0}^{2 \pi}\left|\sin \frac{\sigma}{2}\right|^{1-a}\left\{e^{-h(\sigma)}-4^{1+a} \lambda^{2}\left|\sin \frac{\sigma}{2}\right|^{2 a} \cos ^{2}\left(\frac{\sigma}{2}-\tau\right) e^{h(\sigma)}\right\} d \sigma} .
$$

Since $h(\omega)$ is connected with $f(\omega)$ by equation (8.8), and the parameters $\tau$ and $\lambda$ are given by (8.6), (8.12), this is an integral equation for the function

(14) See, for instance, [17, Chap. VII]. 
$f(\omega)$. The collection of formulas $(8.6),(8.7),(8.8),(8.12),(8.15)$ will be referred to as the integral equation $\mathbf{A}_{1}^{\prime \prime}$.

9. Equivalence of the integral equation, the mapping problem, and the boundary value problem. We have seen in $\$ \$ 6$ and 7 that (under Assumptions $\alpha, \beta, \gamma)$ a solution of boundary value problem $\mathbf{A}_{1}$ also yields a solution of the following mapping problem.

Problem $\mathrm{A}_{1}^{\prime}$. To determine a positive constant $C$, a real constant $\tau$, and an analytic function $w^{*}(\zeta),|\zeta|>1$, satisfying the following conditions: (i) $w^{*}(\zeta)$ admits the representation (7.9), $\chi(\zeta)$ being continuous for $|\zeta|=1$. (ii) $w^{*}(\infty)>0$ and $\max \left|w_{i}^{*}(\zeta)\right|=\mu$, where $\mu$ is given by (8.11). (iii) If $F(\zeta)$ is given by (5.3), then (5.9) represents a homeomorphism of $|\zeta| \geqq 1$ onto $P+\mathcal{E}(\mathbb{P})$ which takes $\zeta=\infty$ into $z=\infty, \zeta=1$ into $z=z_{T}$.

In the preceding section we have shown that a solution of $\mathbf{A}_{1}^{\prime}$ also yields a solution of the integral equation $\mathbf{A}_{1}^{\prime \prime}$. We shall show now that a solution of $\mathbf{A}_{1}^{\prime \prime}$ yields solutions of $\mathbf{A}_{1}^{\prime}$ and $\mathbf{A}_{1}$.

Assume first that a (continuously differentiable) solution $f(\omega)$ of $\mathbf{A}_{1}^{\prime \prime}$ is given and determine $\lambda, C$, and $\tau$ by (8.12), (8.14), (8.6), respectively. From the way in which the integral equation has been set up, it follows that there exists an analytic function $w^{*}(\zeta)$ such that $w^{*}\left(e^{i \omega}\right)=q^{*}(\omega) e^{-i \theta(\omega)}$, where $q^{*}$ and $\theta$ are given by $(8.1),(8.10)$, and (7.7), respectively. It is also clear that this function will have the properties (i) and (ii) as well as the following (iii'): if $F(\zeta)$ is given by (5.3), and if the constant of integration in (5.9) is chosen properly, then (5.9) maps $|\zeta|=1$ topologically onto $\mathcal{P}$, taking $\zeta=1$ into $z_{T}$. It remains to verify property (iii).

We note first that since the integrands in (5.9) are regular analytic functions in $1<|\zeta|<\infty$ and since by (iii')

$$
\int_{|\zeta|=1} d z=0
$$

(5.9) defines a single-valued mapping of $|\zeta|>1$. From the fact that $w^{*}(\infty)$ $>0$, it follows that $\zeta \rightarrow \infty$ implies that $z \rightarrow \infty$. Assertion (iii) would therefore follow from (iii') if we knew that the mapping (5.9) is locally topological. To verify this we consider the Jacobian of this transformation. It is given by

$$
\frac{\partial(x, y)}{\partial(\xi, \eta)}=\frac{1}{4}\left|F^{\prime}(\zeta)\right|^{2}\left\{\frac{1}{\left|w^{*}(\zeta)\right|^{2}}-\left|w^{*}(\zeta)\right|^{2}\right\} .
$$

Since, by (8.10), (8.11), and (8.12), $\left|w^{*}(\zeta)\right|<1$, the Jacobian never vanishes in $|\zeta|>1$ and the equivalence proof of $\mathbf{A}_{1}^{\prime \prime}$ and $\mathbf{A}_{1}^{\prime}$ is complete.

Assume now that a solution of the mapping problem $\mathbf{A}_{1}^{\prime}$ is given. $B y$ virtue of the mapping, $\phi=\operatorname{Re} F(\zeta)$ may be considered as a function of $(x, y)$ defined in $\mathcal{E}(\mathcal{P})$. This function admits the parametric representation

$$
x=\operatorname{Re} G_{1}(\zeta), \quad y=\operatorname{Re} G_{2}(\zeta), \quad \phi=\operatorname{Re} F(\zeta)
$$


where $G_{1}$ and $G_{2}$ are defined by (7.2). Since $G_{1}^{\prime 2}+{G_{2}^{\prime 2}}^{2}{F^{\prime}}^{2}=0$, (9.1) is a Weierstrass parametric representation of a minimal surface $\left({ }^{15}\right)$, so that $\phi(x, y)$ satisfies equation (1.1). A straightforward computation shows that $\psi=\operatorname{Im} F$ and $w^{*}$ are connected with $\phi(x, y)$ by the relations (1.5), (5.8). Since $\psi$ vanishes for $|\zeta|=1$ boundary condition $\mathfrak{A}$ is satisfied, and it follows from (7.3) that $z_{T}$ is a branchpoint of the level-curve $\psi=0 . w=\phi_{x}-i \phi_{y}$ is continuous on $P$, for $w^{*}(\zeta)$ is on $|\zeta|=1$; $w$ is positive at $z=\infty$, for $w^{*}(\infty)$ is. Finally, since $\mu$ is given by (8.11), $|w|$ attains the maximum $q_{\max }$. Thus all conditions of Problem $\mathbf{A}_{1}$ are satisfied.

10. Integral equations for other boundary value problems. In exactly the same way the boundary value problems $\mathbf{A}_{2}-\mathbf{B}_{3}$ may be reduced to integral equations.

In the case of Problem $\mathbf{A}_{2}$, for instance, we must have

$$
F(\zeta)=C e^{-i \tau}\left(\zeta+\frac{e^{2 i \tau}}{\zeta}\right)
$$

and

$$
w^{*}(\zeta)=\lambda\left(1-\frac{e^{2 i \tau}}{\zeta^{2}}\right) e^{x(\zeta)}
$$

with a real $\tau$ and positive $C$ and $\lambda, \chi(\zeta)$ being subject to the same conditions as before. The resulting integral equation for $f(\omega)$ becomes

$$
f(\omega)=\frac{\int_{0}^{\omega}\left\{e^{-h(\sigma)}-4 \lambda^{2} \sin ^{2}(\omega-\tau) e^{h(\sigma)}\right\} d \sigma}{\frac{1}{2 \pi} \int_{0}^{2 \pi}\left\{e^{-h(\sigma)}-4 \lambda^{2} \sin ^{2}(\omega-\tau) e^{h(\sigma)}\right\} d \sigma},
$$

where $h(\omega)$ is again given by (8.7), (8.8) (with $a=1$ ), $\tau$ is again given by (8.6), and

$$
\lambda=\mu / 2 \max \left\{|\sin (\omega-\tau)| e^{h(\omega)}\right\} .
$$

For Problem $\mathbf{A}_{3}$ we have

$$
F(\zeta)=-i C \log \zeta, w^{*}(\zeta)=\lambda e^{\chi(\zeta)} / \zeta
$$

and the resulting integral equation reads

$$
f(\omega)=\frac{\int_{0}^{\omega}\left\{e^{-h(\sigma)}-\lambda^{2} e^{h(\sigma)}\right\} d \sigma}{\frac{1}{2 \pi} \int_{0}^{2 \pi}\left\{e^{-h(\sigma)}-\lambda^{2} e^{h(\sigma)}\right\} d \sigma}
$$

(15) See, for instance, [7, p. 134]. 
where $h(\omega)$ is given by (8.7) and (8.8) (with $a=1$ ) and

$$
\lambda=\mu / \max e^{h(\omega)} \text {. }
$$

The discussion of Problems $\mathbf{B}_{1}-\mathbf{B}_{3}$ may be left to the reader. We note only the result: the integral equation for a problem $\mathbf{B}_{j}(j=1,2,3)$ is obtained from that for the Problem $\mathbf{A}_{j}$ by replacing $\lambda^{2}$ by $-\lambda^{2}$.

The proof that equation $\mathbf{A}_{1}^{\prime \prime}$ possesses a solution, which will be given in the next chapter, holds, with minor and obvious modifications, for the other integral equations.

REMARK. It is easy to verify (following the reasoning of $\$ 5$ ) that if we set in our integral equations $\lambda=0$ we obtain the integral equation satisfied by the function $f(\omega)$ which describes the correspondence between points of $|\zeta|=1$ and of $P$ in a conformal mapping of $|\zeta|>1$ onto the domain exterior to $P$. This should have been expected, since $\lambda=0$ corresponds to $\mu=0$, that is, to $\max \left(\phi_{x}^{2}+\phi_{y}^{2}\right)=0$, and for "infinitely small" values of $\phi_{x}$ and $\phi_{y}$ equation (1.1) coincides with the Laplace equation.

\section{Solution OF THE INTEgRAL EQUATION}

11. The operator $\mathbf{T}$. We proceed to show that equation $\mathbf{A}_{1}^{\prime \prime}$ (that is, equation (8.15) together with the auxiliary equations (8.6), (8.7), (8.8), and (8.12)) has a solution. Throughout this chapter the function $\Theta(s)$ (satisfying all conditions of $\$ 4$ ) is kept fixed and so are the parameters $a$ and $\mu$. We recall that $0 \leqq a \leqq 1,0<\mu<1$.

It will be convenient to transform equation $\mathbf{A}_{1}^{\prime \prime}$ into an integral equation for the derivative $g(\omega)=f^{\prime}(\omega)$.

Let $\mathbf{L}_{p}(p \geqq 1)$ denote the Banach space of measurable functions $g(\omega)$, $0 \leqq \omega \leqq 2 \pi$, for which the norm

$$
\|g\|_{p}=\left\{\int_{0}^{2 \pi}|g(\omega)| p d \omega\right\}^{1 / p}
$$

is finite. If $g(\omega)$ is continuous, we set $\|g\|_{\infty}=\max |g(\omega)|$. For every $g \in L_{1}$ with $\|g\|_{1}>0$ we define

$$
\begin{gathered}
g^{*}(\omega)=\left(2 \pi /\|g\|_{1}\right) \int_{0}^{\omega}|g(\sigma)| d \sigma, \\
h_{g}(\omega)=\frac{1}{2 \pi} \int_{0}^{\pi}\left\{\Lambda_{\theta^{*}}(\omega+t)-\Lambda_{\theta}^{*}(\omega-t)\right\} \cot \frac{t}{2} d t,
\end{gathered}
$$

where $\Lambda_{g} *$ is the function defined by (8.7) with $f=g^{*}$,

$$
\tau(g)=\frac{3 \pi}{2}-\frac{1}{2 \pi} \int_{0}^{2 \pi} \Theta\left[g^{*}(\omega)\right] d \omega,
$$




$$
\begin{aligned}
Q_{g}(\omega) & =2^{1+a}\left|\sin \frac{\omega}{2}\right|^{a}\left|\cos \left[\frac{\omega}{2}-\tau(g)\right]\right| e^{h_{g}(\omega)}, \\
\lambda(g) & =\mu /\left\|Q_{g}\right\|_{\infty},
\end{aligned}
$$

and

$$
R_{g}(\omega)=\left|\sin \frac{\omega}{2}\right|^{1-a} e^{-h_{g}(\omega)}\left\{1-\lambda(g)^{2} Q_{g}(\omega)^{2}\right\} .
$$

Finally we define the operator $l=\mathbf{T}(g)$ by the equation

$$
l(\omega)=\left(2 \pi /\left\|R_{g}\right\|_{1}\right) R_{g}(\omega) .
$$

Lemma 11.1. If $g \in \mathbf{L}_{p}(p>1),\|g\|_{1}>0$, then $\mathbf{T}(g)$ is defined and $l(\omega)$ $=\mathbf{T}(g(\omega))$ is a continuous non-negative function. Furthermore

$$
\|T(g)\|_{1}=2 \pi \text {. }
$$

Proof. For every $g \in \mathbf{L}_{p} \subset \mathbf{L}_{1},\|g\|_{1}>0, g^{*}(\omega)$ is absolutely continuous and the derivative

$$
g^{* \prime}(\omega)=d g^{*}(\omega) / d \omega
$$

belongs to $\mathbf{L}_{p}$. It follows from Hölder's inequality that $g^{*}(\omega)$ satisfies a Hölder condition. Since $\Theta(s)$ is assumed to satisfy a Hölder condition, the function $\Theta\left[g^{*}(\omega)\right]$ satisfies a Hölder condition. Since we have that $g^{*}(0)=0, g^{*}(2 \pi)$ $=2 \pi$, the function $\Lambda_{g^{*}}(\omega)$ also satisfies a Hölder condition. It follows from Privaloff's theorem (see $\$ 13$ ) that the function $h_{g}(\omega)$ conjugate to $-\Lambda_{g^{*}}$ is continuous. Hence $Q_{g}$ is continuous, $\lambda(g)$ may be defined, and $R_{g}$ as well as $\mathrm{T}(g)$ are continuous functions. The fact that $R_{g}(\omega) \geqq 0$ and $\left\|R_{g}\right\|_{1}>0$ follows at once from the definition of $\lambda(g)$.

LEMMA 11.2. Let $f(\omega)$ be a continuously differentiable solution of equation $\mathbf{A}_{1}^{\prime \prime}$. Then $g(\omega)=f^{\prime}(\omega)$ satisfies the equation

$$
g=\mathbf{T}(g) \text {. }
$$

Conversely, if $g \in \mathbf{L}_{p}, p>1$, satisfies equation (11.9), then

$$
f(\omega)=\int_{0}^{\omega} g(\sigma) d \sigma
$$

is a continuously differentiable solution of $\mathbf{A}_{1}^{\prime \prime}$.

The proof may be left to the reader.

12. Application of the Leray-Schauder theorem. We shall base our proof of the solvability of (11.9) on a general existence theorem due to Leray and Schauder which we restate in a form convenient for our purposes $\left({ }^{16}\right)$.

(16) See [19], in particular p. 63. 
Theorem of Leray-Schauder. Let $\Omega$ be a bounded domain (open connected set) of a separable real Banach space $\mathbf{B}, \Omega^{\prime}$ the boundary of $\Omega, \mathbf{F}(k, g)$ an operator defined in $\bar{\Omega}=\Omega+\Omega^{\prime}$ with values in $\mathbf{B}$ and depending upon a real parameter $k, 0 \leqq k \leqq 1$. If for $0 \leqq k \leqq 1, g \in \bar{\Omega}$,

(A) the operator $\mathbf{F}(k, g)$ is jointly uniformly continuous in $k$ and $g$, and

(B) completely continuous in $g$, if

(C) for no $k, 0 \leqq k \leqq 1$, does the equation

$$
g=\mathbf{F}(k, g)
$$

possess a solution on the boundary $\Omega^{\prime}$ and if

(D) the equation (12.1) possesses a solution in $\Omega$ for $k=0$, the mapping

$$
f=g-\mathbf{F}(0, g)
$$

being a homeomorphism, then equation (12.1) possesses a solution in $\Omega$ for every $k, 0 \leqq k \leqq 1$.

Condition (A) means that

$$
\left\|\mathbf{F}(k, g)-\mathbf{F}\left(k^{\prime}, g^{\prime}\right)\right\|<\eta\left(\left\|g-g^{\prime}\right\|+\left|k-k^{\prime}\right|\right), \quad \quad \lim _{\epsilon \rightarrow 0} \eta(\epsilon)=0 .
$$

Condition (B) means that the closure of the image of $\bar{\Omega}$ under the mapping $\mathbf{F}(k, g)$ is compact.

In order to apply this theorem to equation (11.9), we set

$$
\mathbf{F}(k, g)=(1-k)+k \mathbf{T}(g)
$$

so that for $k=1$, equation (12.1) becomes identical with (11.9). For B we take the space $L_{p}, p>1$, for $\Omega$ the domain $\Omega_{\delta}$ determined (for a sufficiently small positive $\delta$ ) by the inequalities

$$
\|g\|_{1}>\delta, \quad\|g\|_{p}<1 / \delta .
$$

We shall prove two statements concerning the operator $\mathbf{T}$.

(I) For every $p>1$ and every sufficiently small $\delta>0, T$ is a uniformly and completely continuous operator defined in $\bar{\Omega}_{\delta}$.

(II) For an appropriately chosen $p>1$ there exists a constant $C$ such that for every $g \in \mathbf{L}_{p},\|g\|_{1}>0$,

$$
\|\mathbf{T}(g)\|_{p}<C
$$

Statement (I) implies at once that conditions (A) and (B) are satisfied. Statement (II) implies that condition (C) is satisfied (for the appropriately chosen $p$ ) for a sufficiently small $\delta$, since by virtue of (11.9) we have that $\|\mathbf{F}(k, g)\|_{1}=2 \pi$ for every $g$ for which $\mathbf{T}(g)$ is defined. Condition (D), finally, is satisfied trivially for every sufficiently small $\delta$, since now equation (12.1) for $k=0$ has the solution $g(\omega) \equiv 1$ and the mapping (12.2) is in our case a simple translation: $f(\omega)=g(\omega)-1$. 
Thus the solvability of (11.9) will be verified once we prove (I) and (II).

13. Theorems on conjugate functions. The essential step in constructing $\mathbf{T}(g)$ for a given $g$ consists in forming a conjugate function. If $\sigma(\omega)$ is periodic with period $2 \pi$ and integrable, then the conjugate function $\bar{\sigma}(\omega)$ is defined by the almost everywhere convergent integral

$$
\bar{\sigma}(\omega)=-\frac{1}{2 \pi} \int_{0}^{\pi}[\sigma(\omega+t)-\sigma(\omega-t)] \cot \frac{t}{2} d t .
$$

Thus relation (11.2) may be written in the form

$$
-h_{g}=\bar{\Lambda}_{g^{*}}
$$

We list now some theorems (either known or trivial) which will be used in the sequel. $\operatorname{By} \mathbf{H}(\alpha, A)$ we denote here and hereafter the class of functions satisfying the Hölder condition

$$
\left|f(\omega)-f\left(\omega^{\prime}\right)\right| \leqq A\left|\omega-\omega^{\prime}\right| \alpha, \quad 0 \leqq \omega \leqq \omega^{\prime} \leqq 2 \pi .
$$

(i) If $\sigma \in \mathbf{H}\left(\alpha, A_{1}\right)$, then $|\bar{\sigma}| \leqq A_{2} A_{1}$, where $A_{2}$ depends only on $\alpha$.

The proof is clear.

(ii) Privaloff's theorem( $\left.{ }^{17}\right)$. If $\sigma \in \mathbf{H}\left(\alpha, A_{1}\right), 0<\alpha<1$, then $\bar{\sigma} \in \mathbf{H}\left(\alpha, A_{3}\right)$ where $A_{3}$ depends only on $\alpha$ and $A_{1}$.

(iii) M. Riesz's Theorem( $\left.{ }^{18}\right)$. If $\sigma \in \mathbf{L}_{p}, p>1$, then $\bar{\sigma} \in \mathbf{L}_{p}$, and $\|\bar{\sigma}\|_{p}$ $\leqq A_{4}\|\sigma\|_{p}$ where $A_{4}$ depends only on $p$.

(iv) Zygmund's Theorem( $\left.{ }^{19}\right)$. If $|\sigma|<A_{5}<\pi / 2$, then

$$
\int_{0}^{2 \pi} e^{\bar{\sigma}(\omega)} d \omega \leqq 2 \pi \sec A_{5}
$$

(v) If $\sigma(\omega)$ is absolutely continuous and $\sigma^{\prime}(\omega) \geqq-A_{6}, A_{6}>0$, then $\bar{\sigma}(\omega)$ $\leqq A_{6} \log 4$.

The proof follows at once from the relation

$$
\bar{\sigma}(\omega)=\frac{1}{2 \pi} \int_{0}^{2 \pi} \sigma^{\prime}(t) \log \sin ^{2} \frac{t-\omega}{2} d t
$$

which is obtained from (13.1) for almost every $\omega$ by integration by parts.

We also note that the relation

$$
\int_{0}^{2 \pi} \bar{\sigma}(\omega) d \omega=0
$$

$\left.{ }^{17}\right)[13]$. See also $\left[17\right.$, p. 156]. The statement on $A_{3}$ while not explicitly stated there follows from the proof.

(18) [14]. See also [17, pp. 147-149].

(19) [18]. Cf. equation (6) on p. 286. 
holds for every $\sigma$.

14. Uniform and complete continuity of T. In this section $p$ and $\delta$ are fixed numbers, $p>1, \delta>0$, and so small that inequalities (12.4) determine a domain $\Omega_{\delta}$. By $B_{i}$ we denote positive constants and by $\eta_{i}(\epsilon)$ positive functions such that $\eta_{i}(\epsilon) \rightarrow 0$ as $\epsilon \rightarrow 0$. Both the $B_{i}$ and the $\eta_{i}$ will, in general, depend on $p$ and $\delta$. All inequalities involving the $B_{i}$ and $\eta_{i}$ are understood to hold in $\bar{\Omega}_{\delta}$. By $q$ we denote the "complementary exponent" of $p: p^{-1}+q^{-1}=1$.

We shall have occasion to use the following almost self-evident lemma.

LEMмA 14.1. Let $\mathcal{C}$ be a class of equicontinuous functions defined over the interval $(0,2 \pi)$. There exists a function $\eta(p, \epsilon)$ such that for $p \geqq 1$ and $f(\omega) \in \mathcal{C}$,

$$
\|f\|_{\infty} \leqq \eta\left(p,\|f\|_{p}\right), \quad \lim _{\epsilon \rightarrow 0} \eta(p, \epsilon)=0 .
$$

We now recall the definition (11.1)-(11.7) of the operator $T$ and note first that by Hölder's inequality

$$
\|g\|_{1} \leqq(2 \pi)^{1 / q}\|g\|_{p}
$$

Using this inequality we conclude easily that

$$
\left\|g_{1}^{*}-g_{2}^{*}\right\|_{\infty} \leqq B_{1}\left\|g_{1}-g_{2}\right\|_{p}
$$

Also

$$
\left\|g^{* \prime}\right\|_{p} \leqq B_{2}
$$

so that by Hölder's inequality

$$
g^{*} \in \mathbf{H}\left(1 / q, B_{3}\right) .
$$

Since $0 \leqq g^{*}(\omega) \leqq 2 \pi$ for $0 \leqq \omega \leqq 2 \pi$, it follows from (4.2) and (14.2) that

$$
\left|\Theta\left[g^{*}(\omega)\right]-\Theta\left[g^{*}\left(\omega^{\prime}\right)\right]\right| \leqq B_{4}\left|\omega-\omega^{\prime}\right|^{\alpha}, \quad 0 \leqq \omega \leqq 2 \pi,
$$

where

$$
\alpha=k / q, \quad 0<\alpha<1 .
$$

Since $g^{*}(0)=0, g^{*}(2 \pi)=2 \pi$, it follows from the definition of $\Lambda_{f}(\mathrm{cf}$. (8.7)) that

$$
\Lambda_{g^{*}} \in \mathbf{H}\left(\alpha, B_{5}\right),
$$

so that by (13.2), Theorem (i) of $\$ 13$, and Privaloff's theorem (ii),

$$
\begin{gathered}
\left\|h_{g}\right\|_{\infty} \leqq B_{6}, \\
h_{g} \in \mathbf{H}\left(\alpha, B_{7}\right) .
\end{gathered}
$$

The last relation implies that

$$
\left(h_{g_{1}}-h_{g_{2}}\right) \in \mathbf{H}\left(\alpha, 2 B_{7}\right)
$$


and applying Lemma 14.1 we see that

$$
\left\|h_{\sigma_{1}}-h_{\sigma_{2}}\right\|_{\infty} \leqq \eta_{1}\left(\left\|h_{\sigma_{1}}-h_{\sigma_{2}}\right\|_{p}\right) .
$$

Now, $h_{\theta_{1}}-h_{\mathbf{g}_{2}}$ is conjugate to the function $\Xi(\omega)$ defined by

$$
\begin{aligned}
& \Xi(\omega)=\Theta\left[g_{2}^{*}(\omega)\right]-\Theta\left[g_{1}^{*}(\omega)\right], \\
& 0 \leqq \omega \leqq 2 \pi, \\
& \Xi(\omega+2 \pi)=\Xi(\omega) .
\end{aligned}
$$

From (14.1) and condition $2^{\circ}, \S 4$, we conclude that

$$
\|\Xi\|_{\infty} \leqq \eta_{2}\left(\left\|g_{1}-g_{2}\right\|_{p}\right),
$$

so that

$$
\|\Xi\|_{1}, \quad\|\Xi\|_{p} \leqq \eta_{3}\left(\left\|g_{1}-g_{2}\right\|_{p}\right) .
$$

From Riesz's theorem it follows that

$$
\left\|h_{o_{1}}-h_{o_{2}}\right\|_{p} \leqq \eta_{4}\left(\left\|g_{1}-g_{2}\right\|_{p}\right),
$$

so that by (14.5)

$$
\left\|h_{g_{1}}-h_{g_{2}}\right\|_{\infty} \leqq \eta_{5}\left(\left\|g_{1}-g_{2}\right\|_{p}\right) .
$$

Also, since $\left|\tau\left(g_{1}\right)-\tau\left(g_{2}\right)\right| \leqq(1 / 2 \pi)\|\Xi\|_{1}$,

$$
\left|\tau\left(g_{1}\right)-\tau\left(g_{2}\right)\right| \leqq \eta_{6}\left(\left\|g_{1}-g_{2}\right\|_{p}\right) .
$$

By (14.8), (14.13), (14.9) we have that

$$
\left\|Q_{g}\right\|_{\infty} \leqq B_{8}, \quad\left\|Q_{o_{1}}-Q_{g_{2}}\right\|_{\infty} \leqq \eta_{7}\left(\left\|g_{1}-g_{2}\right\|_{p}\right),
$$

so that

$$
\begin{array}{r}
\left|\lambda\left(g_{1}\right)-\lambda\left(g_{2}\right)\right| \leqq \eta_{8}\left(\left\|g_{1}-g_{2}\right\|_{p}\right), \\
\quad\left\|R_{o_{1}}-R_{g_{2}}\right\|_{\infty} \leqq \eta_{9}\left(\left\|g_{1}-g_{2}\right\|_{p}\right) .
\end{array}
$$

From the definition of $\lambda(g)$ we have the obvious inequality

$$
1-\mu^{2} \leqq R_{g}(\omega) /\left\{\left|\sin \frac{\omega}{2}\right|^{1-a} e^{-h_{g}(\omega)}\right\} \leqq 1,
$$

which, together with (14.3), (14.8), (14.12), implies that

$$
\left\|R_{\theta}\right\|_{1} \geqq 1 / B_{9}, \quad\left\|R_{\theta}\right\|_{\infty} \leqq B_{10}, \quad\left\|R_{g_{1}}-R_{\theta_{2}}\right\|_{1} \leqq \eta_{10}\left(\left\|g_{1}-g_{2}\right\|_{p}\right) .
$$

The inequalities (14.12), (14.14) imply that

$$
\left\|\mathbf{T}\left(g_{1}\right)-\mathbf{T}\left(g_{2}\right)\right\|_{p} \leqq \eta_{11}\left(\left\|g_{1}-g_{2}\right\|_{p}\right)
$$

so that $\mathbf{T}$ is uniformly continuous in $\bar{\Omega}_{\delta}$.

From the inequalities (14.4), (14.10), (14.14), we obtain that 


$$
\|\mathbf{T}(g)\|_{\infty} \leqq B_{11}, \quad\left|\mathbf{T}(g(\omega))-\mathbf{T}\left(g\left(\omega^{\prime}\right)\right)\right| \leqq \eta_{12}\left(\left|\omega-\omega^{\prime}\right|\right) .
$$

Thus the functions $\{\mathbf{T}(g)\}, g \in \bar{\Omega}_{\delta}$, form a uniformly bounded, equicontinuous family. By Ascoli's theorem the closure of $\mathbf{T}\left(\bar{\Omega}_{\delta}\right)$ is compact in the Banach space of continuous functions, hence, a fortiori, compact in $\mathbf{L}_{p}$. Thus $\mathbf{T}$ is completely continuous in $\bar{\Omega}_{\delta}$ and statement (I) of $\$ 12$ is verified.

15. Estimate of $T(g)$. We proceed to verify statement (II) of $\$ 12$. At first we choose a fixed value $p$ such that

$$
p>1, \quad p \int_{0}^{2 \pi}\left|\min \left[\Theta^{\prime}(\sigma), 0\right]\right| d \sigma<\pi .
$$

This is possible since we assumed the profile $\mathcal{P}$ to be "essentially convex" (cf. (4.3)). By $C_{i}$ we shall denote positive constants which depend upon the value of $p$ chosen above, but not upon $\delta$. All inequalities involving the $C_{i}$ are assumed to hold in every $\bar{\Omega}_{\delta} \subset \mathbf{L}_{p}$.

Now set

$$
\begin{aligned}
\Theta_{+}(s) & =\Theta(0)+\int_{0}^{s} \max \left[\Theta^{\prime}(\sigma), 0\right] d \sigma, \\
\Theta(s) & =\Theta_{+}(s)-\Theta_{-}(s)
\end{aligned}
$$

and for some fixed $g \in \mathbf{L}_{p},\|g\|_{p}>0$,

$$
\begin{array}{rlrl}
L_{1}(\omega) & =\Theta_{+}\left[g^{*}(\omega)\right]-(1 / 2 \pi)\left[\Theta_{+}(2 \pi)-\Theta(0)\right] \omega, & 0 \leqq \omega \leqq 2 \pi, \\
L_{2}(\omega) & =\Theta_{-}\left[g^{*}(\omega)\right]-(1 / 2 \pi) \Theta_{-}(2 \pi) \omega, & & 0 \leqq \omega \leqq 2 \pi, \\
L_{1}(\omega+2 \pi) & =L_{1}(\omega), \quad L_{2}(\omega+2 \pi)=L_{2}(\omega) . & &
\end{array}
$$

Then

$$
L_{1}(\omega)-L_{2}(\omega)=\Lambda_{\sigma^{*}}(\omega)
$$

so that if we define

$$
H_{1}=-\bar{L}_{1}, \quad H_{2}=-\bar{L}_{2}
$$

we have that

$$
H_{1}(\omega)-H_{2}(\omega)=h_{0}(\omega) .
$$

Let $m$ and $M$ be the minimum and maximum of $L_{2}(\omega)$, respectively. Since $\Theta_{-}\left[g^{*}(\omega)\right]$ is monotonic it follows easily that

$$
M-m \leqq \Theta_{-}(2 \pi)=C_{1}<\pi .
$$

Set $L_{3}(\omega)=L_{2}(\omega)-(M+m) / 2$. Then $\left|L_{3}(\omega)\right| \leqq C_{1} / 2$, and

$$
\left|p L_{3}(\omega)\right| \leqq C_{2}<\pi / 2
$$

by virtue of (15.1). Since $H_{2}=-\bar{L}_{2}=-\bar{L}_{3}$, we have by Zygmund's theorem 
$(\$ 13,($ iv) ) that

$$
\int_{0}^{2 \pi} e^{p\left|H_{2}(\omega)\right|} d \omega \leqq C_{3}
$$

A fortiori,

$$
\int_{0}^{2 \pi}\left|\sin \frac{\omega}{2}\right|^{(1-a) p} e^{p H_{2}(\omega)} d \omega \leqq C_{3} .
$$

On the other hand $L_{1}(\omega)$ is absolutely continuous and since, for $0 \leqq \omega \leqq 2 \pi$,

$$
L_{1}^{\prime}(\omega)=\Theta_{+}^{\prime}\left[g^{*}(\omega)\right] g^{* \prime}(\omega)-(1 / 2 \pi)\left[\Theta_{+}(2 \pi)-\Theta(0)\right],
$$

we have, noting that $\Theta_{+}^{\prime} \geqq 0, g^{* \prime} \geqq 0$,

$$
L_{1}^{\prime}(\omega) \geqq-C_{4}<0 .
$$

Thus by Theorem (v) of $\$ 13$,

$$
-H_{1}(\omega) \leqq C_{5}, \quad e^{-p H_{1}(\omega)} \leqq C_{6},
$$

so that by (15.2) and (15.3),

$$
\int_{0}^{2 \pi}\left|\sin \frac{\omega}{2}\right|^{(1-a) p} e^{-p h_{g}(\omega)} d \omega \leqq C_{7} .
$$

By virtue of (14.13) this inequality implies that

$$
\left\|R_{\theta}\right\|_{p} \leqq C_{8}
$$

Finally we apply the integral form of the inequality between the arithmetic and the geometric means,

$$
\frac{1}{2 \pi} \int_{0}^{2 \pi} \chi(\omega) d \omega \geqq \exp \left\{\frac{1}{2 \pi} \int_{0}^{2 \pi} \log \chi(\omega) d \omega\right\}, \quad \chi(\omega) \geqq 0,
$$

to the function $\chi(\omega)=[\sin (\omega / 2)]^{1-a} e^{-h_{g}(\omega)}$. Since the mean-value of $h_{g}$ vanishes (cf. $\$ 13$, in particular (13.3)), we see that

$$
\frac{1}{2 \pi} \int_{0}^{2 \pi}\left|\sin \frac{\omega}{2}\right|^{1-a} e^{-h_{g}(\omega)} d \omega \geqq 2^{a-1}=1 / C_{10} .
$$

Together with (14.13) this implies that

so that by (15.14)

$$
\left\|R_{0}\right\|_{1} \geqq 1 / C_{11} \text {, }
$$

$$
\|\mathbf{T}(g)\|_{p} \leqq C_{12} .
$$

Thus statement (II) is proved and so is our existence theorem. 


\section{Extensions}

16. A modification of Problem $\mathbf{A}_{1}$. The aerodynamical problem of finding a circulation free Chaplygin flow past a profile with a sharp trailing edge leads to the following modification of Problem $\mathbf{A}_{1}$.

Problem $\mathbf{A}_{4}$. The profile $\mathcal{P}$ and the function $\phi$ are to satisfy the same conditions as in Problem $\mathbf{A}_{1}$, except that condition $w_{\infty}>0$ is replaced by the requirement that $\phi(x, y)$ be single-valued. have

Repeating the reasoning of Chapter II we see that in this case we must

$$
\begin{aligned}
F & =C\left(1-\frac{1}{\zeta^{2}}\right) \\
w^{*} & =\lambda e^{i \alpha}\left(1-\frac{1}{\zeta}\right)^{a}\left(1+\frac{1}{\zeta}\right) e^{x(\zeta)}, \quad e^{x(\infty)}=1 .
\end{aligned}
$$

For the function $f(\omega)$ we obtain the integral equation

$$
f(\omega)=\frac{\int_{0}^{\omega}\left|\sin \frac{\sigma}{2}\right|^{1-a}\left\{e^{-h(\sigma)}-4^{1+a} \lambda^{2}\left|\sin \frac{\sigma}{2}\right|^{2 a} \cos ^{2} \frac{\sigma}{2} e^{h(\sigma)}\right\} d \sigma}{\frac{1}{2 \pi} \int_{0}^{2 \pi}\left|\sin \frac{\sigma}{2}\right|^{1-a}\left\{e^{-h(\sigma)}-4^{1+a} \lambda^{2}\left|\sin \frac{\sigma}{2}\right|^{2 a} \cos ^{2} \frac{\sigma}{2} e^{h(\sigma)}\right\} d \sigma}
$$

where $h$ and $\lambda$ are given again by (8.7), (8.8), and (8.12) (in the last formula one must set $\tau=0)$. For $\alpha$ we obtain an equation involving $f(\omega)$ which we have no need to write down explicitly. The existence proof for equation (16.1) follows at once from the considerations of the preceding chapter.

We leave to the reader formulation of the problems "dual" to $\mathbf{A}_{4}$ and to the problem $\mathbf{A}_{5}$ to be discussed in the next section.

17. Profiles with two sharp corners. If our profile possesses two sharp corners or cusps $z_{T}$ and $z_{L}$, we can formulate a problem analogous to Problem $\mathbf{A}_{1}$, by replacing the condition $w_{\infty}>0$ by the "Kutta-Joukowski condition" relative to $z_{L}$.

We assume now that $P$ admits the representation

$$
z=Z(s)=z_{T}+\int_{0}^{s} \exp \left\{i\left[\Theta(\sigma)+\Theta_{1}(\sigma)\right]\right\} d \sigma, \quad 0 \leqq \sigma \leqq 2 \pi,
$$

where

$$
\begin{gathered}
\Theta_{1}(s)=0, \quad 0<s<s_{1}, \quad \Theta_{1}(s)=(1-b) \pi, \quad s_{1}<s<2 \pi, \\
\Theta(2 \pi)-\Theta(0)=(a+b) \pi, \\
0 \leqq b \leqq 1, \quad 0 \leqq a \leqq 1,
\end{gathered}
$$

and $\Theta(s)$ satisfies conditions $2^{\circ}$ and $3^{\circ}$ of $\S 4$. Thus $z_{L}=Z\left(s_{1}\right)$.

Problem $\mathbf{A}_{5}$. $P$ has no protruding corners or cusps except perhaps two at 
$z_{T}$ and $z_{L}$. These points are branch-points of the level-line $\psi=0$.

Proceeding as in Chapter II we see that $F(\zeta)$ will again be given by (5.3) with

$$
f(\tau)=s_{1},
$$

where $f$ has the same meaning as before, and that

$$
w^{*}(\zeta)=\lambda e^{i \alpha}\left(1-\frac{1}{\zeta}\right)^{a}\left(1+\frac{e^{2 i \pi}}{\zeta}\right)^{b} e^{\chi(\zeta)},
$$

where $\lambda>0, \alpha>0$, and $\chi(\zeta)$ is subject to the same conditions as before.

For the function $f(\omega)$ we obtain the integral equation

(17.2) $f(\omega)=\frac{\int_{0}^{\omega}\left|\sin \frac{\sigma}{2}\right|^{1-a}\left|\cos \left(\frac{\sigma}{2}-\tau\right)\right|^{1-b}\left\{e^{-h(\sigma)}-4^{a+b} \lambda^{2}\left|\sin \frac{\sigma}{2}\right|^{2 a}\left|\cos \left(\frac{\sigma}{2}-\tau\right)\right|^{2 b} e^{h(\sigma)}\right\} d}{\frac{1}{2 \pi} \int_{0}^{2 \pi}\left|\sin \frac{\sigma}{2}\right|^{1-a}\left|\cos \left(\frac{\sigma}{2}-\tau\right)\right|^{1-b}\left\{e^{-h(\sigma)}-4^{a+b} \lambda^{2}\left|\sin \frac{\sigma}{2}\right|^{2 a}\left|\cos \left(\frac{\sigma}{2}-\tau\right)\right|^{2 b} e^{h(\sigma)}\right\} d}$

where $\tau$ is given by (17.1), $h(\omega)$ is again given by equations (8.7), (8.8) (with $(1+a)$ in $(8.7)$ replaced by $(a+b))$, and

$$
\lambda=\mu / \max \left\{2^{a+b}\left|\sin \frac{\omega}{2}\right|^{a}\left|\cos \left(\frac{\omega}{2}-\tau\right)\right|^{b} e^{h(\omega)}\right\} .
$$

For $\alpha$ we obtain easily an expression involving $f(\omega)$ which we have no need to write down explicitly.

In order to prove the solvability of (17.2), we define, for every $g \in \mathbf{L}_{p}$, $p>1,\|g\|_{1}>0$, and for every real number $\tau \in \mathbf{E}_{1}\left(\mathbf{E}_{n}\right.$ denotes here and hereafter the Euclidean $n$-space), the following operators and functionals:

$$
Q_{o, \tau}(\omega)=2^{a+b}\left|\sin \frac{\omega}{2}\right|^{a}\left|\cos \left(\frac{\omega}{2}-\tau\right)\right|^{b} e^{h_{g}(\omega)}
$$

where $h_{g}$ is again defined by (11.1), (11.2),

$$
\begin{aligned}
\lambda(g, \tau) & =\mu /\left\|Q_{g, \tau}\right\|_{1}, \\
R_{g, \tau}(\omega) & =\left|\sin \frac{\omega}{2}\right|^{1-a}\left|\cos \left(\frac{\omega}{2}-\tau\right)\right|^{1-b} e^{-h_{g}(\omega)}\left\{1-\lambda(g, \tau)^{2} Q_{g, \tau}(\omega)^{2}\right\} .
\end{aligned}
$$

We also define, for every strictly increasing function $f(\omega)$ such that $f(0)=0$, $f(2 \pi)=2 \pi$, the number $\tilde{t}(f)$ by the equation

$$
f[\tilde{t}(f)]=s_{1} .
$$

Proceeding as in $\$ 14$ we can show that for $g \in \bar{\Omega}_{\delta}$ inequality (14.3) holds, and that we have (analogously to (14.13))

$$
1-\mu^{2} \leqq R_{\theta, \tau}(\omega) /\left\{\left|\sin \frac{\omega}{2}\right|^{1-a}\left|\cos \left(\frac{\omega}{2}-\tau\right)\right|^{1-b} e^{-h_{g}(\omega)}\right\} \leqq 1 .
$$

This implies that 


$$
1 / B_{12} \leqq R_{g, \tau}(\omega)\left|\sin \frac{\omega}{2}\right|^{-1+a}\left|\cos \left(\frac{\omega}{2}-\tau\right)\right|^{-1+b} \leqq B_{12}
$$

where $B_{12}$ is a constant depending on $\delta$ and $p$.

If we define the operator $l=\mathrm{T}_{\tau}(\mathrm{g})$ by equation (11.7), and set

$$
\tilde{g}_{\tau}=l^{*}=\mathbf{T}_{\tau}(g)^{*}, \quad \text { that is, } \tilde{g}(\omega)=\int_{0}^{\omega} l(\sigma) d \sigma
$$

(where the *-operation is defined by (11.1)), then it follows from (17.4) that $\tilde{g}$ is strictly increasing. Obviously $g(0)=0, g(2 \pi)=2 \pi$, so that the functional

$$
\mathbf{t}_{\tau}(g)=\tilde{t}(\tilde{g})
$$

is well-defined.

It is immediately clear that our integral equation (17.2) is equivalent to the following system of equations for the function $g(\omega)=f^{\prime}(\omega)$ and the number $\tau$ :

$$
g=\mathbf{T}_{\tau}(g), \quad \tau=\mathbf{t}_{\tau}(g) .
$$

Now let $\mathbf{E}_{1} \times \mathbf{L}_{p}$ be the Banach space of pairs $(\tau, g), \tau \in \mathbf{E}_{1}, g \in \mathbf{L}_{p}$, with the obvious definition of addition and multiplication by scalars, and with the norm:

$$
\||(\tau, g)|\|=\left\{|\tau|^{2}+\|g\|_{p}^{2}\right\}^{1 / 2} .
$$

We define (for $\|g\|_{p}>0$ ) the operator $\mathbf{T}$ by

$$
\mathbf{T}(\tau, g)=\left(\mathbf{t}_{\tau}(g), \mathbf{T}_{\tau}(g)\right)
$$

and write (17.6) in the form

$$
(\tau, g)=\mathbf{T}(\tau, g) .
$$

We also introduce in (17.7) a real parameter $k, 0 \leqq k \leqq 1$, setting

$$
\mathbf{F}(k ; \tau, g)=(1-k)(0,1)+k \mathbf{T}(\tau, g) .
$$

Using the methods of Chapter III we can show that $\mathrm{F}(k ; \tau, g)$ satisfies all conditions of the Schauder-Leray theorem with respect to a domain $(0,2 \pi) \times \Omega_{\delta} \subset \mathbf{E}_{1} \times \mathbf{L}_{p}$, for appropriately chosen $\delta$ and $p$.

A new argument is needed only for the verification of the uniform continuity of $t_{\tau}(g)$ for

$$
0 \leqq \tau \leqq 2 \pi, \quad g \in \bar{\Omega}_{\delta} \subset \mathbf{L}_{p} \quad(p>1) .
$$

We shall sketch this argument briefly.

At first, reasoning as in $\$ 14$, we conclude that

$$
\left\|\mathbf{T}_{\tau_{1}}\left(g_{1}\right)-\mathbf{T}_{\tau_{2}}\left(g_{2}\right)\right\| \leqq \eta_{13}\left(\left|\left\|\left(\tau_{1}, g_{1}\right)-\left(\tau_{2}, g_{2}\right) \mid\right\|\right),\right.
$$


where $\eta_{13}$ is a function of the kind used in $\$ 14$. We already noted that (14.3) and (17.3) hold under the hypotheses (17.7). Therefore, setting $l=\mathbf{T}_{\tau}(g)$, we have

$$
l(\omega) \geqq\left(1 / B_{13}\right)\left|\sin \frac{\omega}{2}\right|^{1-a}\left|\cos \left(\frac{\omega}{2}-\tau\right)\right|^{1-b} .
$$

Set

$$
\left(\tau_{i}^{\prime}, l_{i}\right)=\mathbf{T}\left(\tau_{i}, g_{i}\right), \quad i=1,2 .
$$

Then

$$
\int_{0}^{\tau_{1}^{\prime}} l_{1}(\omega) d \omega=\int_{0}^{\tau_{2}^{\prime}} l_{2}(\omega) d \omega
$$

so that

$$
\left|\int_{\tau_{1}^{\prime}}^{\tau_{2}^{\prime}} l_{2}(\omega) d \omega\right| \leqq \int_{0}^{\tau_{1}^{\prime}}\left|l_{1}(\omega)-l_{2}(\omega)\right| d \omega \leqq B_{14}\left\|\mathbf{T}_{\tau_{1}}\left(g_{1}\right)-\mathbf{T}_{\tau_{2}}\left(g_{2}\right)\right\|_{p} .
$$

On the other hand there exists a strictly increasing $\Delta(\epsilon)$ such that for $\omega \neq \omega^{\prime}$ and all $\tau$,

$$
\left.\left|\int_{\omega}^{\omega^{\prime}}\right| \sin \frac{\sigma}{2}\right|^{1-a}\left|\cos \left(\frac{\sigma}{2}-\tau\right)\right|^{1-b} d \sigma \mid \geqq \Delta\left(\left|\omega-\omega^{\prime}\right|\right)>0 .
$$

If $\left(\tau_{1}, g_{1}\right)$ and $\left(\tau_{2}, g_{2}\right)$ belong to the closure of $(0,2 \pi) \times \Omega_{\delta}$, we have by (17.11) and (17.9) that

$$
\left|\int_{\tau_{1}^{\prime}}^{\tau_{2}^{\prime}} l_{2}(\omega) d \omega\right| \leqq \eta_{14}\left(||\left|\left(\tau_{1}-\tau_{2}, g_{1}-g_{2}\right)\right|||\right),
$$

and by (17.10) and (17.12) that

$$
\left|\int_{\tau_{1}^{\prime}}^{\tau_{2}^{\prime}} l(\omega) d \omega\right| \leqq\left(1 / B_{14}\right) \Delta\left(\left|\tau_{1}^{\prime}-\tau_{2}^{\prime}\right|\right) .
$$

From (17.13) and (17.14) it follows that

$$
\left|\tau_{1}^{\prime}-\tau_{2}^{\prime}\right| \leqq \eta_{15}\left(\left|\left\|\left(\tau_{1}-\tau_{2}, g_{1}-g_{2}\right) \mid\right\|\right),\right.
$$

that is, the uniform continuity of $t_{\tau}(g)$.

18. Profiles with intruding corners. The preceding results remain true if we permit profiles $P$ possessing a finite number of intruding corners, provided that we change suitably the definition of "essential convexity." More precisely, we may replace the hypotheses stated in $\$ 4$ by the following $\left({ }^{20}\right)$.

${ }^{(20)}$ For the sake of simplicity we assume that $z_{T}$ is not the vertex of an intruding corner. 
The profile $P$ admits the representation

$$
z=z_{T}+\int_{0}^{s} \cdot \exp \left\{i\left[\Theta(\sigma)+\Theta_{1}(\sigma)\right]\right\} d \sigma, \quad 0 \leqq s \leqq 2 \pi,
$$

where

$$
\begin{array}{crr}
\Theta_{1}(\sigma) & =\pi \sum_{\nu=1}^{N} c_{\nu} \vartheta\left(s-s_{\nu}\right), & 0<s_{1}<s_{2}<\cdots<s_{N}<2 \pi, \\
c_{\nu} & \geqq 0, & \vartheta(s)=0 \text { for } s<0, \quad \vartheta(s)=-1 \text { for } s>0,
\end{array}
$$

and the following conditions are satisfied.

$1^{\circ}$. Set $\Theta(2 \pi)-\pi\left(c_{1}+c_{2}+\cdots+c_{N}\right)-\Theta(0)=(1+a) \pi$. Then $a=1$, except in Problems $\mathbf{A}_{1}, \mathbf{B}_{1}$, where $0 \leqq a \leqq 1$.

$2^{\circ}$. The function $\Theta(s)$ satisfies a uniform Hölder condition.

$3^{\circ}$ (Essential convexity). $\Theta(s)$ is absolutely continuous and

$$
\pi \sum_{\nu=1}^{N} c_{\nu}+\int_{0}^{2 \pi}\left|\min \left[\Theta^{\prime}(\sigma), 0\right]\right| d \sigma<\pi .
$$

The integral equation, for Problem $\mathbf{A}_{3}$ say $\left({ }^{21}\right)$, now becomes

$$
f(\omega)=\frac{\int_{0}^{\omega} \prod_{\nu=1}^{N}\left|\sin \frac{\sigma-\tau_{\nu}}{2}\right|^{-c_{\nu}}\left\{e^{-h(\sigma)}-4^{2 \gamma} \lambda^{2} \prod_{\nu=1}^{N}\left|\sin \frac{\sigma-\tau_{\nu}}{2}\right|^{2 c_{\nu}} e^{h(\sigma)}\right\} d \sigma}{\frac{1}{2 \pi} \int_{0}^{2 \pi} \prod_{\nu=1}^{N}\left|\sin \frac{\sigma-\tau_{\nu}}{2}\right|^{-c_{\nu}}\left\{e^{-h(\sigma)}-4^{2 \gamma} \lambda^{2} \prod_{\nu=1}^{N}\left|\sin \frac{\sigma-\tau_{\nu}}{2}\right|^{2 c_{\nu}} e^{h(\sigma)}\right\} d \sigma}
$$

where

$$
\gamma=\sum_{\nu=1}^{N} c_{\nu}
$$

$h(\omega)$ is given by the same formulae as before (with $(1+a)$ in (8.7) replaced by $(1+a+\gamma))$,

$$
\lambda=\mu / \max \left\{2^{\gamma} \prod_{\nu=1}^{N}\left|\sin \frac{\omega-\tau_{\nu}}{2}\right|^{c_{\nu}} e^{h(\sigma)}\right\},
$$

and the $\tau_{\nu}$ are determined by the equations $f\left(\tau_{\nu}\right)=s_{\nu}, \nu=1,2, \cdots, N$. The existence proof for equation (18.2) can be carried out by the methods already described.

We shall now have to consider the Banach space $\mathbf{E}_{N} \times \mathrm{L}_{p}$ of elements $\left(\tau_{1}, \tau_{2}, \cdots, \tau_{N}, g\right)$ with $\tau_{j} \in \mathbf{E}_{1}, g \in \mathbf{L}_{p}$ and the norm

$$
\|\|\left(\tau_{1}, \cdots, \tau_{N}, g\right) \mid \|=\left\{\sum_{j=1}^{N}\left|\tau_{j}\right|^{2}+\|g\|_{p}^{2}\right\}^{1 / 2} .
$$

(21) We consider this problem instead of Problem $\mathbf{A}_{1}$, say, only in order to obtain a less cumbersome integral equation. 
After defining an appropriate operator $\mathbf{T}$ :

$$
\left(\tau_{1}^{\prime}, \tau_{2}^{\prime}, \cdots, \tau_{N}^{\prime}, l\right)=\mathbf{T}\left(\tau_{1}, \tau_{2}, \cdots, \tau_{N}, g\right)
$$

we shall be able to write the equation satisfied by the derivative $g(\omega)=f^{\prime}(\omega)$ of a solution of (18.2) in the form

$$
\left(\tau_{1}, \cdots, \tau_{N}, g\right)=\mathbf{T}\left(\tau_{1}, \cdots, \tau_{N}, g\right) .
$$

We may leave it to the reader to carry out for this equation the considera tion analogous to those of Chapter III and \$17. A new argument will be needed only for verifying the complete continuity of $\mathbf{T}$.

In fact, a function $l(\omega)$ defined by (18.4) will not be continuous any more. Instead it will be of the form

$$
l(\omega)=r(\omega) \prod_{\nu=1}^{N}\left|\sin \frac{\omega-\tau_{\nu}^{\prime}}{2}\right|^{-c_{\nu}}
$$

with a continuous $r(\omega)$. It will turn out, however, that if $\left(\tau_{1}, \cdots, \tau_{N}, g\right)$ is restricted to the closed region

$$
0 \leqq \tau_{\nu} \leqq 2 \pi, \quad \nu=1,2, \cdots, N, \quad g \in \overline{\Omega_{\delta}},
$$

the corresponding functions $r(\omega)$ will form a uniformly bounded equicontinuous family. From this the complete continuity of $\mathbf{T}$ will follow easily.

We also note that throughout the whole proof one would have to make use of the inequality $\gamma<1$ which follows at once from (18.1) and (18.3).

19. Open questions. The following problems are suggested by the preceding results:

1. Do the boundary value problems $\mathbf{A}_{i}, \mathbf{B}_{i}(i=1,2,4,5)$ have a solution if we prescribe not the value of $q_{\max }=\max |w|$ but the value of $\left|w_{\infty}\right|$ (cf. (2.1))? In gas-dynamical language: may we prescribe the speed at infinity instead of the maximum local speed $\left({ }^{22}\right)$ ?

From equations (5.8) and (7.9) it follows that prescribing the value of $\left|w_{\infty}\right|$ amounts to considering the parameter $\lambda$ in our integral equations as a given constant (subject to the condition $0<\lambda<1$ ). For Problem $\mathbf{A}_{1}$ it follows from the results of Frankl and Keldysh( $\left.{ }^{5}\right)$ that a solution exists for $0<\lambda<\epsilon$, where $\epsilon$ is a sufficiently small number which may depend upon the profile. The important question, however, is whether or not the same is true for $\epsilon=1$.

2. Are the solutions whose existence has been established unique?

3. Can equation (8.15) or the other integral equations be solved by the

${ }^{(22)}$ From the point of view of gas-dynamics it might be preferable to prescribe $q_{\max }$. In fact, if we work with the differential equation resulting from the actual pressure-density relation and prescribe a (subsonic) value of $\left|w_{\infty}\right|$, we can not be sure that a purely subsonic flow will exist. 
method of successive approximations? Numerical computations seem to indicate that this is possible in some but not in all cases (cf. [2] and [3]).

4. Can the condition of "essential convexity" imposed on the profile be removed or replaced by a weaker condition?

Of even greater interest would be the extension of our results to more general quasi-linear partial differential equations.

\section{REFERENCES}

1. S. Bartnoff and A. Gelbart, National Advisory Committee for Aeronautics, Technical Note 1171 (1947).

2. L. Bers, National Advisory Committee for Aeronautics, Technical Note 1006 (1946).

3. - - National Advisory Committee for Aeronautics, Technical Note 2056 (1950).

4. —— Proceedings of Symposia in Applied Mathematics, vol. 1, New York, American Mathematical Society, 1949, pp. 41-46.

5. — Ann. of Math. vol. 53 (1951) pp. 364-386.

6. S. A. Chaplygin, Sci. Mem. Moscow Univ. Section of Math. and Phys. vol. 21 (1902) pp. 1-121.

7. R. Courant and D. Hilbert, Methoden der mathematischen Physik, vol. 2. 601.

8. F. Frankl and M. Keldysh, Bull. Acad. Sci. URSS. Sér. Math. vol. 12 (1934) pp. 561-

9. A. Gelbert, National Advisory Committee for Aeronautics, Technical Note 1170 (1947).

10. A. Gelbart and D. Resch, National Advisory Committee for Aeronautics, Technical Note 2057 (1950).

11. A. Hurwitz and R. Courant, Funktionentheorie.

12. J. Leray and J. Schauder, Ann. Ecole Norm. vol. 51 (1934) pp. 46-78.

13. J. Privaloff, Bull. Soc. Math. France vol. 44 (1916) pp. 100-103.

14. M. Riesz, Math. Zeit. vol. 27 (1927) pp. 218-244.

15. N. A. Slioskin, Sci. Mem. Moscow Univ. Section of Math. and Phys. vol. 7 (1937) pp. 43-69.

16. T. W. Chen, Trans. Amer. Math. Soc. vol. 65 (1949) pp. 331-347.

17. A. Zygmund, Trigonometrical series.

18. — , Fund. Math. vol. 13 (1929) pp. 284-303.

SYRACUSE UNIVERSITY,

Syracuse, N. Y. 